\title{
Preparation and Characterization of a New Monoclonal Antibody Specific Against Lawsonia intracellularis and Its Application in Indirect Immunofluorescence and Immunocytochemistry Assay
}

\author{
Ning Xiao ${ }^{1}$, Jiannan $\mathrm{Li}^{1}$, Minxue $\mathrm{Li}^{1}$, Yuting Hu${ }^{1}$, Huixing $\mathrm{Lin}^{1}$ and Hongjie Fan ${ }^{1,2 *}$ \\ ${ }^{1}$ MOE Joint International Research Laboratory of Animal Health and Food Safety, College of Veterinary Medicine, Nanjing \\ Agricultural University, Nanjing, China, ${ }^{2}$ Jiangsu Co-innovation Center for the Prevention and Control of Important Animal \\ Infectious Diseases and Zoonoses, Yangzhou University, Yangzhou, China
}

OPEN ACCESS

Edited by:

Anbu K. Karuppannan, Tamil Nadu Veterinary and Animal

Sciences University, India

Reviewed by:

Carlos Pereira,

Universidade Federal de Viçosa, Brazil Marta Campillo,

University of Glasgow, United Kingdom

*Correspondence:

Hongjie Fan

fhj@njau.edu.cn

Specialty section: This article was submitted to Veterinary Infectious Diseases, a section of the journal

Frontiers in Veterinary Science

Received: 05 August 2021 Accepted: 21 October 2021 Published: 29 November 2021

Citation:

Xiao N, Li J, Li M, Hu Y, Lin H and

Fan $\mathrm{H}$ (2021) Preparation and

Characterization of a New Monoclonal

Antibody Specific Against Lawsonia intracellularis and Its Application in Indirect Immunofluorescence and

Immunocytochemistry Assay.

Front. Vet. Sci. 8:753610

doi: 10.3389/fvets.2021.753610
Proliferative enteropathy (PE) is an infectious enteric disease caused by Lawsonia intracellularis ( $L$. intracellularis) and is endemic in pig herds worldwide. However, a $L$. intracellularis-specific monoclonal antibody plays an important role in the evaluation of $L$. intracellularis infection in vitro. Therefore, the objective of this study was to produce and identify the characteristics of a new monoclonal antibody against the outer membrane protein (Omp2) of $L$. intracellularis and apply it in an indirect immunofluorescence assay (IFA) and immunocytochemistry (IHC). The results indicated that three highly specific monoclonal antibodies against the Omp2 protein (4D9, 3G2, and 7G5) of $L$. intracellularis were obtained by using purified Omp2 as an immunogen, the titers of ascitic fluids of 4D9, 3G2, and 7G5 cells were 1:2,048,000, 1:512,000, and 1:256,000, respectively. IFA analysis showed that the 4D9, 3G2, and $7 \mathrm{G} 5$ have no cross-reactivity with other enteric bacteria commonly found in the ilea of pigs or closely related to $L$. intracellularis, such as Desulfovibrio, Bilophila wadsworthia (B. wadsworthia), Salmonella choleraesuis (S. choleraesuis), Salmonella typhimurium (S. typhimurium), Escherichia coli (E. coli), and Brachyspira hyodysenteriae (B. hyodysenteriae). IFA and IHC results indicated that the monoclonal antibodies can be successfully used as primary antibodies to detect $L$. intracellularis in infected cells and in the crypt of the ileum from infected tissues of PE. Our findings suggested that the new monoclonal antibody specific against $L$. intracellularis will be useful for the evaluation of $L$. intracellularis infection in vivo and in vitro.

Keywords: proliferative enteropathy, Lawsonia intracellularis, Omp2 protein, monoclonal antibody, indirect immunofluorescence assay, immunocytochemistry

\section{INTRODUCTION}

Proliferative enteropathy (PE) is an infectious enteric disease characterized by thickening of the ileal wall as a result of enterocyte proliferation associated with the presence of Lawsonia intracellularis $(1,2)$. L. intracellularis causes PE in various species, such as pigs, horses, hamsters, dogs, and non-human primates (3-6). Porcine 
intestinal adenomatosis (PIA) is considered to be the chronic form of $\mathrm{PE}$, which leads to mild diarrhea and reduced growth in young pigs aged $8-20$ weeks $(7,8)$. Proliferative hemorrhagic enteropathy (PHE), an acute form of $\mathrm{PE}$, often occurs in older finisher pigs, gilts, and sows, is characterized by bloody diarrhea, and often leads to sudden death $(7,8)$.

L. intracellularis has been widely spread in more than 20 countries, such as China, Canada, Brazil, Finland, France, South Africa, Greece, and India (9, 10), and the disease causes more than $\$ 1.63$ per infected pig due to bloody diarrhea, sudden death, decreased body weight, stunted growth, and increased costs of feeding and medication worldwide (11-13). In Denmark, the herd prevalence detected by qPCR and ELISA were 90.0 and $91.7 \%$, while in the United Kingdom, the herd prevalence detected by the commercial kit SVANOVIR ${ }^{\circledR} L$. intracellularis/Ileitis-Ab was $100.0 \%$ (14). In the field of China, the seroprevalence of $L$. intracellularis antibodies in pigs on intensive farms was evaluated by a commercial blocking ELISA kit in 2014 (15), and the overall true prevalence of L. intracellularis animal seropositivity was $77 \%$ (95\% CI, 70-83\%). A higher rate of seroprevalence was found in fattening pigs, sows, and boars than in preweaning piglets and weaners (15). The results indicate that the infection of L. intracellularis is common in pig herds in China. Therefore, to control the infection and spread of PPE effectively, it is crucial to obtain the L. intracellularis strain isolated from China and prepare an effective PPE vaccine, while the prerequisite for successful isolation of $L$. intracellular is to prepare the specific monoclonal antibody to monitor the bacteria in tissue sample and infected cell monolayer.

Only a few laboratories around the world are capable of isolation and cultivation of L. intracellularis due to the fastidious microaerophilic obligate intracellular nature of this organism $(6,16,17)$. To date, there is no information about successful isolation and maintenance of $L$. intracellularis infection in vitro in China. This is mainly because the cells are easily contaminated after being inoculated with the intestinal homogenate of infected pigs as $L$. intracellularis resides in a contaminated environment. Furthermore, sensitive and accurate methods suitable for determination of the viability and detection of $L$. intracellularis directly from the cell culture are scarce. Although a variety of methods are available for evaluation of a highly L. intracellularis-infected cell monolayer when isolating and cultivating $L$. intracellularis from clinical intestinal samples, such as PCR (18), fluorescent in situ hybridization (FISH) (19), and immunoperoxidase monolayer assay (IPMA) (20), these methods also have some limitations. A major limitation of PCR is that it has no ability to differentiate between live and dead bacteria; Furthermore, PCR results only indicate the nucleic acid of infected cells and unable to confirm whether the bacteria has entered the intestinal epithelial cells. Although FISH is well-standardized and easily performed, it requires experience in interpreting the results. A previous study has shown that autofluorescence could produce false-positive results, and the use of PNA probes remains expensive, and the sensitivity of FISH remains lower than PCR in the case of analyses from primary materials $(21,22)$. Moreover, the infected cells also could be detected by IPMA after being incubated for 5 days in a gas concentration of $8.0 \%$ oxygen $\left(\mathrm{O}_{2}\right), 8.8 \%$ carbon dioxide $\left(\mathrm{CO}_{2}\right)$, and $83.2 \%$ nitrogen $\left(\mathrm{N}_{2}\right)$, which is time consuming (23). Therefore, the PCR, FISH, and IPMA methods are not the most effective way to monitor the infected cells when isolating L. intracellularis. However, immunofluorescence assay (IFA) presents many advantages such as repeatable, sensitive, and less time consuming (24). Furthermore, the infected cells could be precisely detected after being incubated for $3 \mathrm{~h}$ in a gas concentration of $8.0 \% \mathrm{O}_{2}, 8.8 \% \mathrm{CO}_{2}$, and $83.2 \% \mathrm{~N}_{2}$, which will improve the detection efficiency when isolating $L$. intracellularis from an infected intestine.

Therefore, the objectives of the present study was to produce and identify the characteristics of a new monoclonal antibody against outer membrane protein (Omp2) of L. intracellularis and apply it in an indirect immunofluorescence assay (IFA) and immunocytochemistry (IHC). Our findings may lay the basis for evaluating the monolayer of McCoy, IEC-18, IPEC-J2, and many other cell lines and tissues infected with $L$. intracellularis.

\section{MATERIALS AND METHODS}

\section{Bacterial Strains, Plasmids, and Cell Lines}

The $L$. intracellularis strain (a commercial live attenuated $L$. intracellularis vaccine, Enterisol ${ }^{\circledR}$ ileitis) was purchased from Boehringer Ingelheim Vetmedica, Germany, and Escherichia coli (CAU0751), Salmonella choleraesuis (CVCC2139), and Salmonella typhimurium (CVCC2220) were purchased from the China Veterinary Culture Collection Center (CVCC). Desulfovibrio (ATCC 27774) and Bilophila wadsworthia (ATCC 49260) were purchased from the American Type Culture Collection (ATCC). Brachyspira hyodysenteriae is courtesy of the Shanghai Animal Disease Prevention and Control Center. The pGex-6p-1 (+) vector was purchased from Novagen, USA.

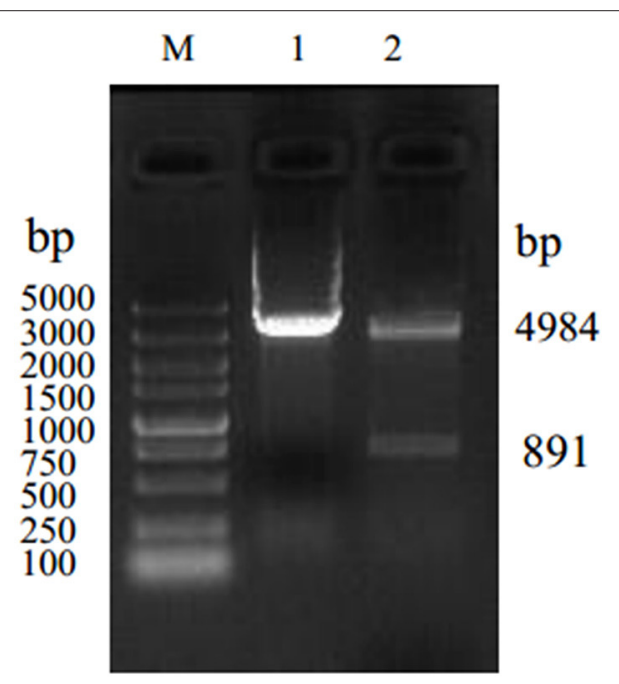

FIGURE 1 | Identification of the recombinant plasmid pGex-6p-1-Omp2 by restriction enzyme digestion with BamH I and Sal I. Lane M. DNA marker 5,000; lane 1: product of plasmid pGex-6p-1; lane 2: recombinant plasmid pGex-6p-1-Omp2. 

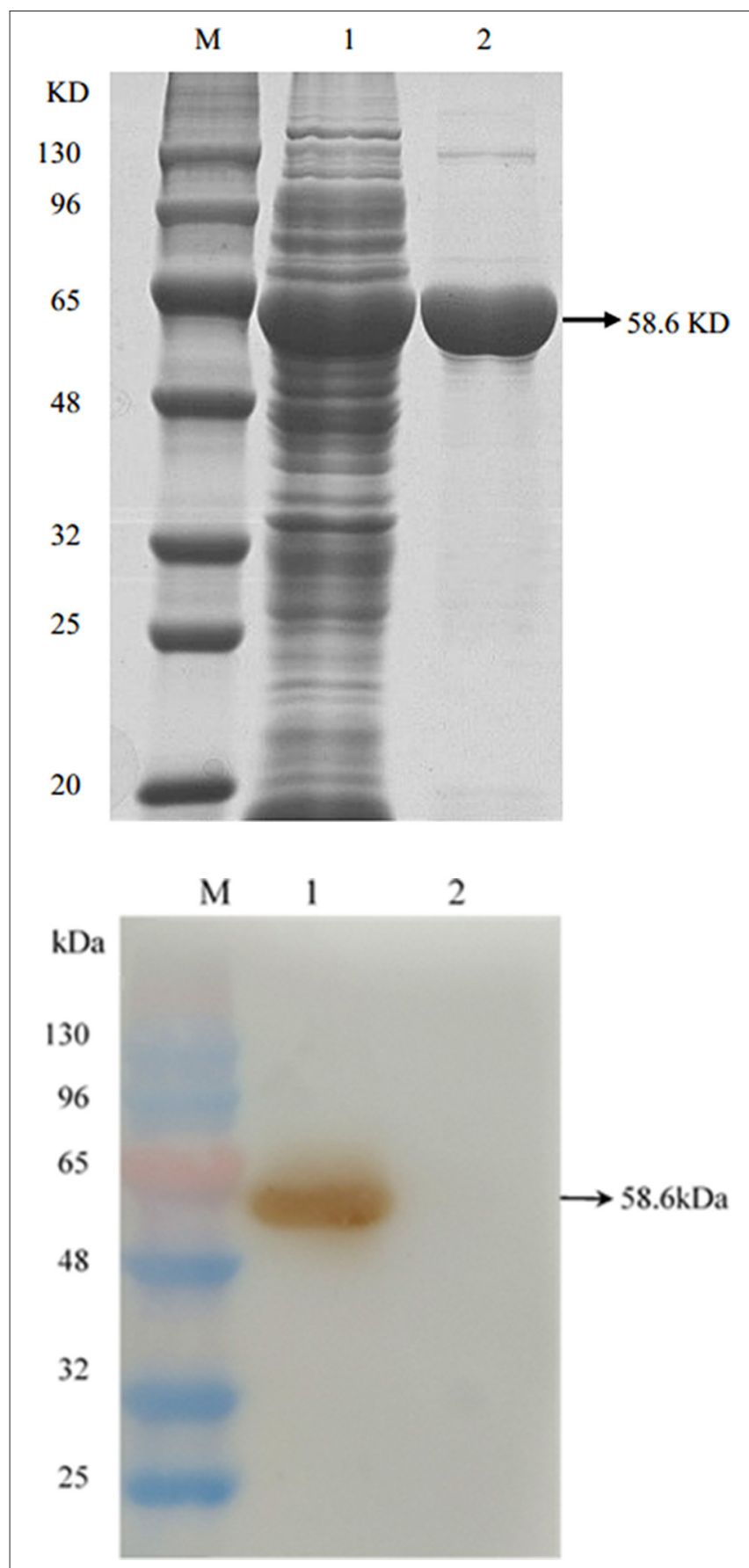

FIGURE 2 | The expression and identification of Omp2 protein. (A) SDS-PAGE analysis of Omp2 expressed in E. coli BL21. Lane M: protein molecular weight standard; lane 1: soluble fraction of the induced bacterial lysate, lane 2: purified Omp2 protein. (B) Western blotting analysis for Omp2. An anti-L. intracellularis-positive antibody served as the primary antibody. Lane M: the prestained protein molecular weight standard; lane 1: purified Omp2; lane 2: purified GST-tagged protein.

The myeloma cell line SP2/0 (stored in our laboratory) and rat small intestine cells (IEC-18; ATCC CRL 1589) were cultured in DMEM/high glucose (Gibco, USA) in a humidified $5 \%$ $\mathrm{CO}_{2}$ atmosphere at $37^{\circ} \mathrm{C}$. All the cell culture media were

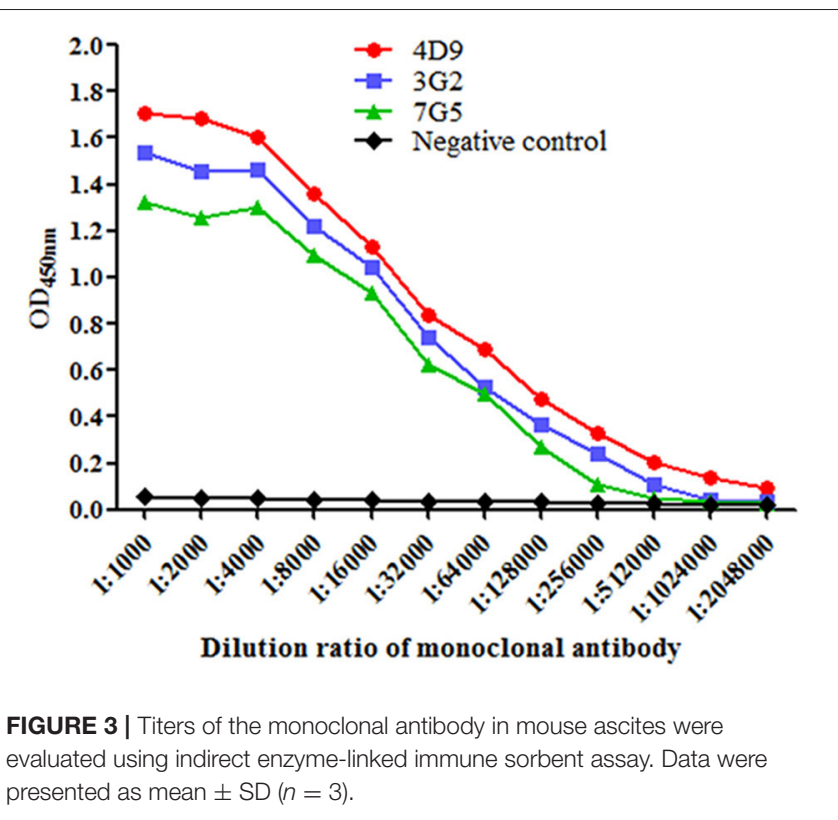

TABLE 1 | Subtype identification of the monoclonal antibodies.

\begin{tabular}{lccc}
\hline Subtype & 4D9 & 3G2 & 7G5 \\
\hline IgA & 0.158 & 0.171 & 0.118 \\
$\operatorname{lgM}$ & 0.194 & 0.128 & 0.199 \\
$\operatorname{lgG1}$ & 2.584 & 2.712 & 2.648 \\
$\operatorname{lgG} 2 \mathrm{a}$ & 0.171 & 0.184 & 0.149 \\
$\operatorname{lgG} 2 \mathrm{~b}$ & 0.109 & 0.101 & 0.127 \\
$\operatorname{lgG} 3$ & 0.124 & 0.146 & 0.106 \\
$\lg -\gamma$ & 0.136 & 0.135 & 0.097 \\
$\lg -\mathrm{k}$ & 1.673 & 1.713 & 1.748 \\
\hline
\end{tabular}

supplemented with $10 \%$ fetal bovine serum (FBS, Gibco, USA). $L$. intracellularis was grown as described (5). In brief, 30\% confluent IEC-18 monolayers were infected with $L$. intracellularis and incubated in an atmosphere of $83.2 \%$ nitrogen $\left(\mathrm{N}_{2}\right), 8.8 \%$ carbon dioxide $\left(\mathrm{CO}_{2}\right)$, and $8 \%$ oxygen $\left(\mathrm{O}_{2}\right)$ for 7 days at $37^{\circ} \mathrm{C}$.

\section{Expression, Purification, and Identification of the Outer Membrane Protein}

The outer membrane protein $(O m p 2)$ gene sequences of $L$. intracellularis were selected and analyzed. The partial Omp2 sequence GXNN strain was synthesized according to the 103-993 bp regions from L. intracellularis (no. EU621796.1) by Sangon Biotech Co., Ltd. (Shanghai, China) using restriction enzymes (BamH I and Sal I). The synthetic Omp2 gene was cloned into the prokaryotic expression vector pGex-6p-1 (+). The positive recombinant plasmids were identified by enzyme digestion and sequencing and then transformed into E. coli Rosetta (DE3) competent cells. The expression of the recombinant Omp2 protein was induced with $0.3 \mathrm{mmol} / \mathrm{L}$ isopropyl- $\beta-\mathrm{D}$ thiogalactopyranoside (IPTG, Sigma, USA) at $16^{\circ} \mathrm{C}$ for $18 \mathrm{~h}$, and the protein was examined by SDS-PAGE. The recombinant 


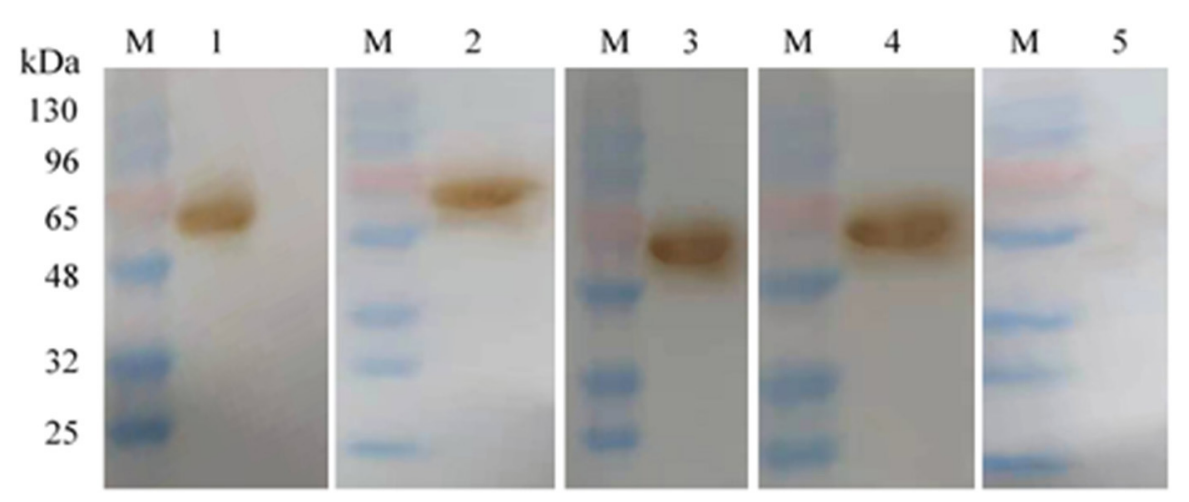

FIGURE 4 | Monoclonal antibody against Omp2 of L. intracellularis was identified by Western blot. Lane M: the prestained protein molecular weight standard; lanes 1-3: 4D9 MAb, 3G2 MAb, and 7G5 MAb, respectively; lane 4: the mouse serum against Omp2 protein; lane 5: unimmunized normal mouse serum.

protein was also detected by SDS-PAGE after purification with a high-affinity GSTRAP HP column (GE Healthcare Life Sciences, USA) according to the instructions of the manufacturer, and the concentration was determined with a NANODROP 2000 Spectrophotometer (Thermo, USA). In addition, Western blotting was performed to evaluate the reactivity of the purified Omp2 protein. The same method was described in the reference with some modifications (25). Briefly, the purified Omp2 protein was transferred onto a polyvinylidene fluoride (PVDF) membrane (Millipore Corporation, USA) after being separated by $12 \%$ SDS-PAGE. Then the membrane was blocked with $5 \%$ skim milk in TBST $(20 \mathrm{mM}$ Tris- $\mathrm{HCl}, 150 \mathrm{mM} \mathrm{NaCl}, 0.01 \%$ Tween-20, pH 7.4) overnight at $4^{\circ} \mathrm{C}$, followed by incubation with an anti- $L$. intracellularis-positive antibody (SVANOVIR ${ }^{\circledR}$ L. intracellularis/Ileitis- $A b$, Germany) for $1 \mathrm{~h}$ at $37^{\circ} \mathrm{C}$. After three washes with TBST, the membrane was incubated with goat anti-pig IgG H\&L (HRP) secondary antibody for $1 \mathrm{~h}$ at $37^{\circ} \mathrm{C}$ (Zhuangmeng, China). After three washes with TBST, the target protein bands were detected using 3,3-diaminobenzidine tetrahydrochloride $(\mathrm{DAB})$, and the reaction was stopped by rinsing with distilled water and drying the membrane.

\section{Production and Identification of Monoclonal Antibodies Against the Outer Membrane Protein}

The animal protocol used was approved by the Ethical Committee of the Faculty of Veterinary Science of Nanjing Agricultural University (approval number: IACUC20181009); and all experiments were performed in accordance with the relevant guidelines and regulations. The purified Omp2 protein was used to immunize 6-week-old BALB/c mice (Yangzhou University Comparative Medical Center, China). The mice were immunized with Freund's adjuvant (Sigma, USA) three times. Three days after the final immunization, the mice with the highest antibody titers were euthanized, and the spleen cells were harvested. Subsequently, splenocytes from immunized mice were fused with $\mathrm{sp} 2 / 0$ murine myeloma cells by using 50\% polyethylene glycol (PEG 4000, Sigma, USA) according to a previously described method (26). The fusion cells were separated into 96-well-plates and cultured selectively in DMEM/high glucose containing 10\% fetal bovine serum, $100 \mathrm{mM}$ hypoxanthine, $400 \mathrm{mM}$ aminopterin, and $16 \mathrm{mM}$ thymidine. On day 5,50 $\mu$ l of HAT medium was added to each well of the 96-cell plates. On day 12, the HAT medium was completely replaced with fresh HT medium. After HAT/HT medium screening, the culture supernatants were analyzed by indirect enzyme-linked immunosorbent assay (ELISA). The positive hybridoma cells were repeatedly subcloned by the limiting dilution method until monoclonal hybridoma cells were obtained. Positive hybridoma cells were cultured in the abdominal cavity of paraffin-primed BALB/c mice to obtain ascitic fluid. The characteristics of the monoclonal antibodies (mAbs) were identified using a Pierce Rapid Isotyping Kit (Thermo Scientific, USA) according to the instructions of the manufacturer.

\section{Enzyme-Linked Immunosorbent Assay}

The antibody titers of ascitic fluid were further determined by ELISA, as previously described (27). Briefly, the purified recombinant Omp2 protein was used as a coating antigen, the ascitic fluids were diluted in serial 2-fold dilutions from 1:1,000 to $1: 2,048,000$ and added to the wells in triplicate, and the negative control (unimmunized normal mouse serum) was included in each plate. After washing five times with PBST (0.5\% Tween-20 in PBS), the plate was incubated with HRP-conjugated goat antimouse IgG (Abmart, China) at a ratio of $1: 5,000$ for $1 \mathrm{~h}$ at $37^{\circ} \mathrm{C}$. The color was developed with 3,3',5,5'- tetramethylbenzidine (TMB), and the reaction was stopped by $2 \mathrm{M} \mathrm{H}_{2} \mathrm{SO}_{4}$. The $\mathrm{OD}_{450 \mathrm{~nm}}$ was measured in a micro titer plate reader (BioRad Benchmark Plus). The cutoff value was determined when the $\mathrm{OD}_{450 \mathrm{~nm}}$ ratio $\left(\mathrm{OD}_{450 \mathrm{~nm}}\right.$ of purified antibody/OD $/ \mathrm{D}_{450 \mathrm{~nm}}$ of unimmunized rabbit serum) was above 2.1. The antibody titer was defined as the highest dilution that yielded a net $\mathrm{OD}_{450 \mathrm{~nm}}$ value greater than the calculated cutoff.

\section{Western Blot Analysis}

The expressed Omp2 proteins were subjected to gel electrophoresis on $12 \%$ SDS-PAGE after denaturation with $2 \times$ SDS loading buffer at $100^{\circ} \mathrm{C}$ for $3 \sim 5 \mathrm{~min}$. The protein bands 


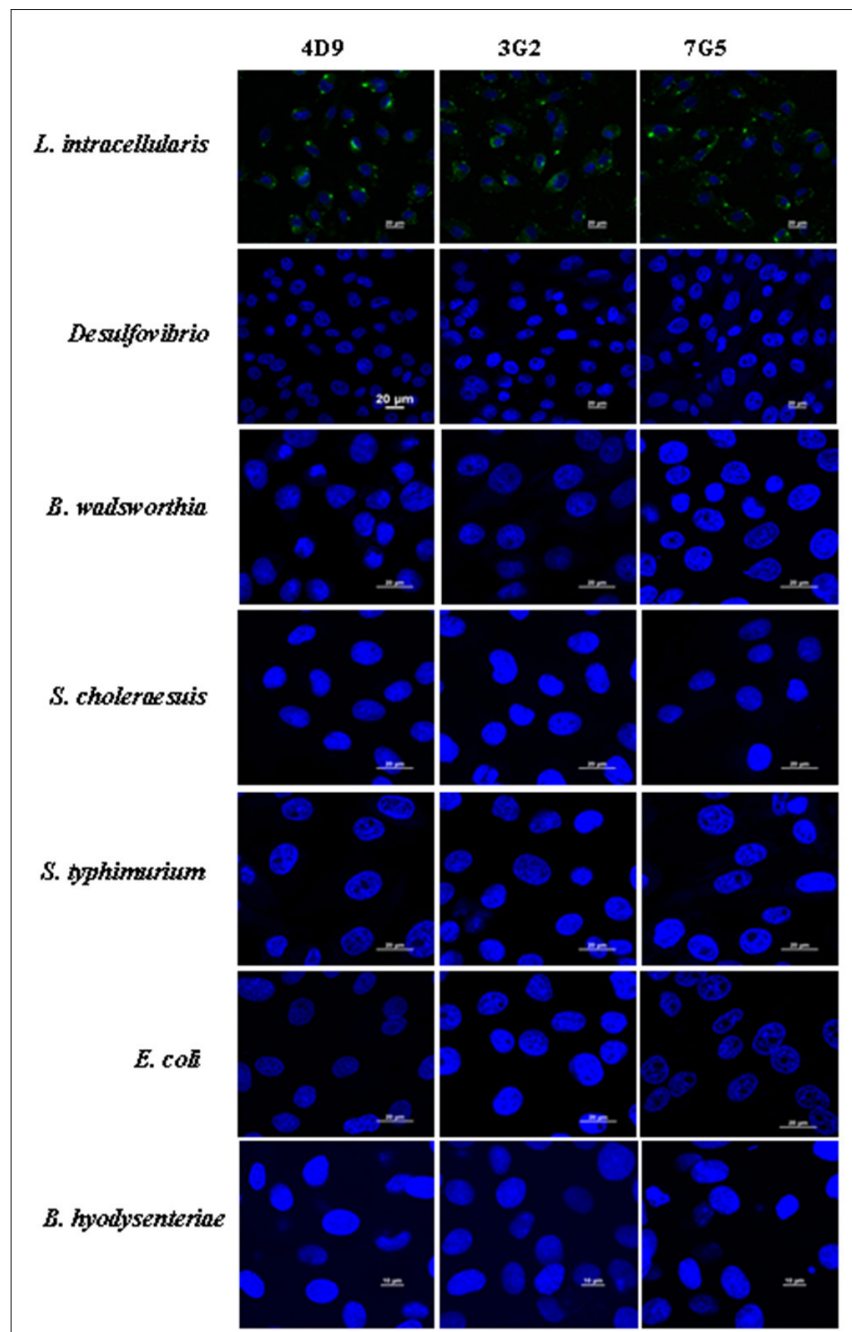

FIGURE 5 | Immunofluorescence results using 4D9 MAb, 3G2 MAb, and 7G5 MAb to evaluate the cross-reactivity with Desulfovibrio, B. wadsworthia, $S$. choleraesuis, S. typhimurium, E. coli, and B. hyodysenteriae after incubated with IEC-18 cells for $3 \mathrm{~h}$

were transferred to PVDF membranes. The membranes were blocked with $5 \%$ skim milk in TBST overnight at $4^{\circ} \mathrm{C}$. After washing three times in TBST, the membranes were incubated with mAbs against Omp2 protein, positive control (mouse serum against Omp2 protein, collected from the last injection) or negative control (unimmunized normal mouse serum) at $37^{\circ} \mathrm{C}$ for $1 \mathrm{~h}$. After washing with TBST, the membranes were immersed in goat anti-mouse HRP-conjugated polyclonal serum at $37^{\circ} \mathrm{C}$ for $1 \mathrm{~h}$. After washing in the same way, the membranes were immersed in $\mathrm{DAB}$, and the reaction was stopped by rinsing with distilled water and drying the membrane.

\section{Assessment of Specificity of Monoclonal Antibodies}

The cross-reactivity of the mAbs was detected by IFA. Briefly, about $1 \times 10^{5}$ L. intracellularis, $1 \times 10^{5} \mathrm{CFU}$ of Desulfovibrio,
B. wadsworthia, S. choleraesuis, S. typhimurium, E. coli, and B. hyodysenteriae were tested using a bacterial suspension to infect IEC-18 cells. The cell cultures were detected after being incubated at $37^{\circ} \mathrm{C}$ in $8.0 \% \mathrm{O}_{2}, 8.8 \% \mathrm{CO}_{2}$, and $83.2 \% \mathrm{~N}_{2}$ in a humidified incubator for $3 \mathrm{~h}$, the rest of the experiment steps were the same as before.

\section{Immunofluorescence and Immunohistochemistry Assay}

Immunofluorescence assays were performed using IEC-18 cells seeded on 13-mm sterilized coverslips and cultured overnight to $30 \%$ confluence. The cells were infected with $1 \times 10^{5} \mathrm{~L}$. intracellularis at a multiplicity of infection (MOI) of 100 for $3 \mathrm{~h}$ and 7 days in a tri-gas incubator with $83.2 \% \mathrm{~N}_{2}, 8.8 \% \mathrm{CO}_{2}$, and $8 \% \mathrm{O}_{2}$ at $37^{\circ} \mathrm{C}$ (Gene Science AG300 plus, USA) according to methods described in the literature (28). Uninfected cells were used as negative control. The cells were washed with PBS three times and fixed with ice-cold $4 \%$ paraformaldehyde $\left(-20^{\circ} \mathrm{C}\right)$ for $15 \mathrm{~min}$, and the cells were permeabilized with $0.3 \%$ TritonX-100 for $10 \mathrm{~min}$ at room temperature. After blocking for $2 \mathrm{~h}$ at $37^{\circ} \mathrm{C}$ in 5\% skim milk in PBS, the fixed cells were then incubated with a 1:200 dilution of $\mathrm{mAbs}$ for $1 \mathrm{~h}$ at $37^{\circ} \mathrm{C}$. Unimmunized normal mouse serum and mouse serum against the Omp2 protein were used as negative and positive controls, respectively. The cell monolayers were rehydrated by rinsing three times with PBST. FITC-labeled goat anti-mouse IgG and goat anti-mouse IgG Alexa Fluor 594 were used as a secondary antibody, respectively, at a $1: 1,000$ dilution and incubated in the dark for $1 \mathrm{~h}$ at $37^{\circ} \mathrm{C}$. After three washes with PBST, $200 \mu \mathrm{l}$ of DAPI (4,6-diamidino2-phenylindole dihydrochloride; Ziyi Reagent Co., Shanghai, China) staining solution was added to each well, and the nuclei were stained in the dark for $10 \mathrm{~min}$ at room temperature. After washing, the coverslips were removed and immediately examined by confocal laser scanning microscope (Nikon ECLIPSE Ti, Japan).

The monoclonal antibody was also evaluated by immunofluorescence (IF) staining and immunocytochemistry (IHC) in histological fragments of lesions suggestive of PPE. Briefly, infected (subclinical form of PE) and normal intestinal samples were obtained from commercial pigs in a slaughterhouse in Jiangsu Province, China. Subsequently, the samples of ileum were fixed by immersion in $10 \%$ neutral-buffered formalin. L. intracellularis-specific Omp2 monoclonal antibody (4D9, 3G2, and 7G5) was used to perform IF and IHC to stain $L$. intracellularis bacteria according to the previously reported methods $(29,30)$. Uninfected sample was used as negative controls at the same time.

\section{RESULTS}

\section{Expression and Purification of the Outer Membrane Protein}

The Omp2 gene was correctly inserted into the pGex-6p1 vector according to nucleotide sequencing and restriction analysis (Figure 1, lane 2). The sequence analysis of the protein 

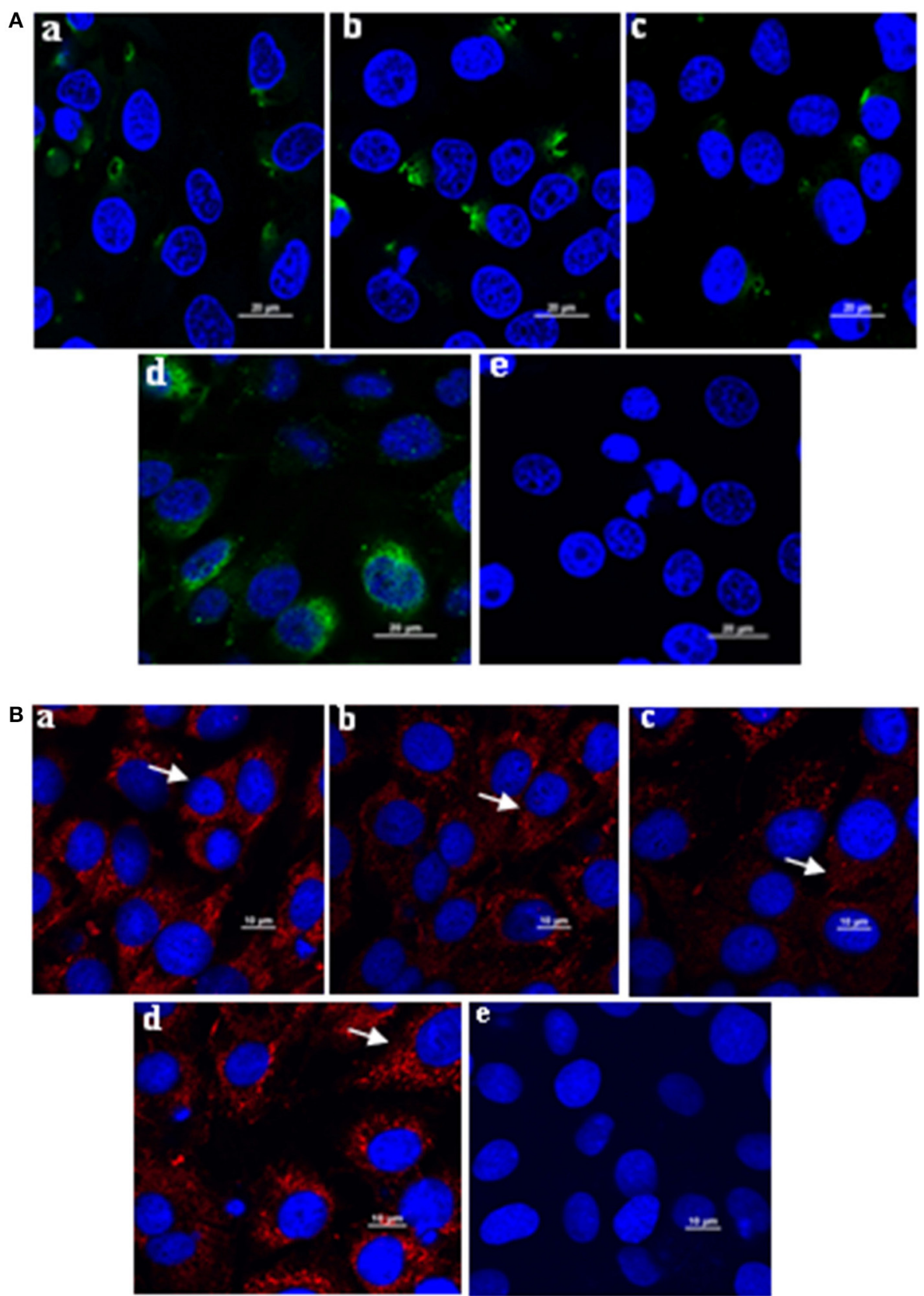

FIGURE 6 | Application of the monoclonal antibody in immunofluorescence assay to monitor the $L$. intracellularis in infected monolayer cells after incubated for $3 \mathrm{~h}$ and 7 days, respectively. (A) L. intracellularis were incubated for $3 \mathrm{~h}$; the presence of $L$. intracellularis by IFA was stained green. (B) $L$. intracellularis were incubated for 7 days, the presence of $L$. intracellularis was stained red. Nuclei were counterstained with DAPI (blue). The mouse serum against Omp2 protein and unimmunized normal mouse serum were used as positive and negative control. (a) 4D9 MAb; (b) 3G2 MAb; (c) 7G5 MAb; (d) positive control; (e) negative control. ×600.

was performed by DNAStar software. MegAlign analysis results showed that the homology of the amino acid sequence between GXNN strain outer membrane protein and LI0902 of PHE/MN100 (GenBank No. AM180252.1) is 99.1\%. Compared with LI0902 of PHE/MN1-00, the 175th (Val to Alu), 223th (Glu to Gly), and 254th (Leu to Ser) amino acids generated mutation amino acids from the GXNN strain. Subsequently, the recombinant
Omp2 protein was successfully expressed in BL21 (DE3) cells after it was induced by IPTG. The expressed recombinant GSTOmp2 protein was $\sim 58.6 \mathrm{kDa}$ and present in the supernatant of bacterial lysates (Figure 2A, lane 1). After purification by a GSTRAP HP column, the purity of the final products in the portion of the eluate was $\sim 98 \%$, as determined by image analysis of Coomassie blue-stained SDS-PAGE (Figure 2A, lane 
2). Western blotting assays showed that the purified Omp2 could be specifically recognized by the anti- $L$. intracellularispositive antibody (Figure 2B, lane 1), whereas no reactive band appeared when purified GST was used as the negative control (Figure 2B, lane 2).

\section{Production of Monoclonal Antibodies Against the Outer Membrane Protein of $L$. intracellularis}

Purified Omp2 proteins were used to immunize BALB/c mice. To avoid obtaining mAbs against the GST tag, the GST tag of Omp2 was digested using PreScission Protease (Beyotime Biotechnology. Inc., China) before it was used as a coating antigen in the ELISA for screening (data not shown). Finally, three hybridoma cell lines named 4D9, 3G2, and 7G5 were acquired by subcloning and screening. The titers of ascitic fluids were measured by indirect ELISA, and the titers of 4D9, 3G2, and 7G5 were 1:2,048,000, 1:512,000 and 1:256,000, respectively (Figure 3).

\section{Subtype Identification of Monoclonal Antibodies}

The results of subtype identification showed that the heavy chains of the three mAbs (4D9, 3G2, and 7G5) all belonged to IgG1, and the light chains were of the kappa type (Table 1).

\section{The Characters of the Monoclonal Antibodies}

Western blotting analysis showed that all the mAbs and the positive control (the mouse serum against Omp2 protein) could react with the Omp2 protein (Figure 4, lane 1-4), whereas no reactive band appeared when the negative control (unimmunized normal mouse serum) was used (Figure 4, lane 5).

\section{Assessment of the Specificity of Monoclonal Antibodies}

To test the specificity of the 4D9, 3G2, and 7G5, the IFA was conducted with pure cultures of the following bacterial strains: Desulfovibrio, B. wadsworthia, S. choleraesuis, $S$. typhimurium, E. coli, and B. hyodysenteriae. The results showed that 4D9, 3G2, and $7 \mathrm{G} 5$ did not have crossreactions with enteric bacteria commonly found in the ileum of pigs or closely related to $L$. intracellularis (Figure 5). The results indicated that the new monoclonal antibody prepared in this study is specific against L. intracellularis.

\section{Application of Monoclonal Antibodies in Immunoflourescense Assay and Immunocytochemistry}

The mAbs could react with $L$. intracellularis in the infected monolayer. In particular, significant specific signals were detected using the primary antibodies against Omp2 (4D9, 3G2, and 7G5). Our findings indicated that the L. intracellularis in the

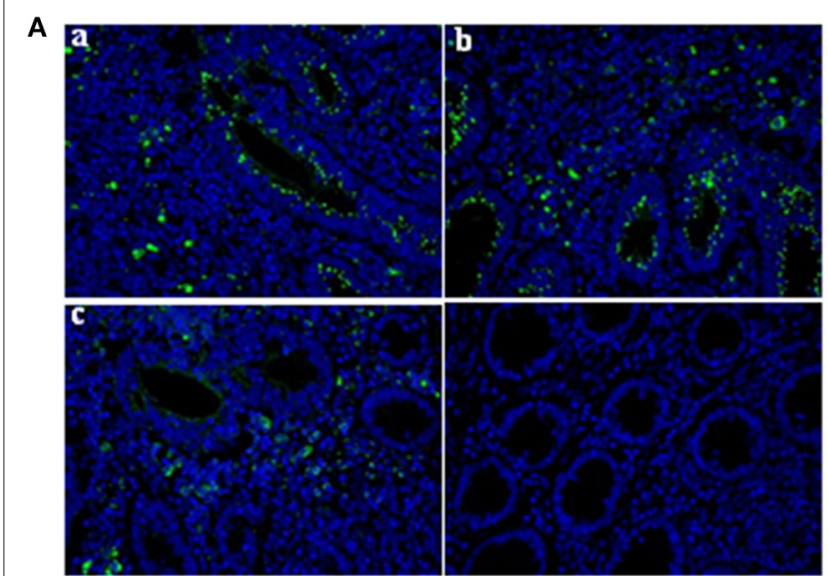

B a

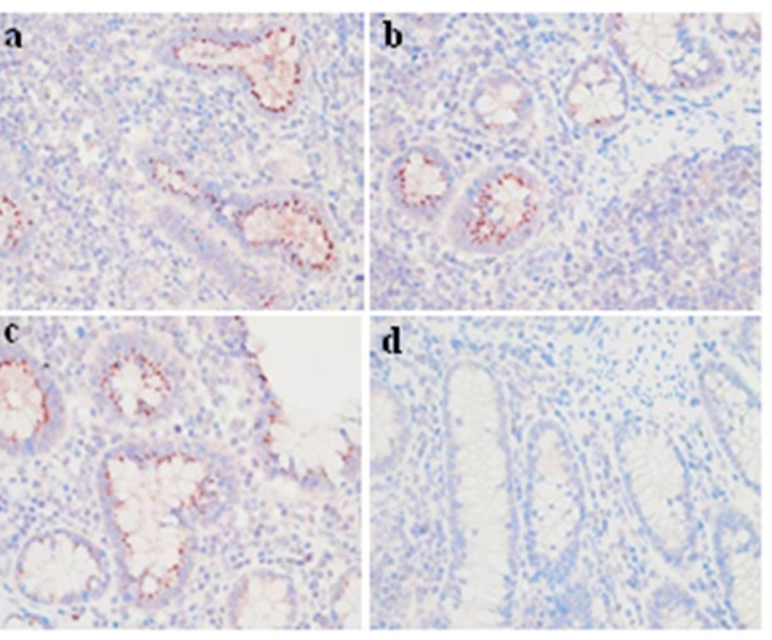

FIGURE 7 | Evaluation of the monoclonal antibody by immunofluorescence and immunocytochemistry in infected tissue of PPE. IF and IHC assays were conducted in a blinded fashion on parallel sections. (A) Immunofluorescence assay. The presence of $L$. intracellularis by IF was detected using $L$. intracellularis-specific monoclonal antibody (4D9, 3G2, and 7G5) (green), nuclei were counterstained with DAPI (blue). (B) Immunocytochemistry assay. The presence of $L$. intracellularis by $\mathrm{HC}$ was detected using $L$.

intracellularis-specific monoclonal antibody (red), nuclei were counterstained with hematoxylin (blue). (a) 4D9 MAb; (b) 3G2 MAb; (c) 7G5 MAb; (d) negative control. $\times 600$.

monolayer of eukaryotic cells could be detected at $3 \mathrm{~h}$ post infection (Figure 6A), and highly infected cells (HIC) may be observed once the bacteria are cultivated for up to 7 days post incubation. However, no signal was detected in the negative control (Figure 6B). IFA and IHC results indicated that $L$. intracellularis antigen was visualized mainly in the crypt of the ileum from infected tissues of PPE after it reacted with $L$. intracellularis-specific monoclonal antibody, while control ileum was negative (Figures 7A,B). These results demonstrated that the new monoclonal antibody could also be applied to evaluate $L$. intracellularis antigen in infected tissues of PPE. 


\section{DISCUSSION}

Monoclonal antibody plays an important role in the isolation and culture of L. intracellularis. In previous studies, the preparation of monoclonal antibodies against $L$. intracellularis mainly focused on LsaA protein and the whole bacteria protein of $L$. intracellularis $(31,32)$. Monoclonal antibody against surface antigens (LsaA) of L. intracellularis has been used as the primary antibody to detect antigen in histological tests and have been successfully detected in various field isolates from affected tissues of pigs and other animals. However, the MAb of LsaA was limited due to the lack of the LsaA gene in the commercial live attenuated $L$. intracellularis vaccine. In our study, the outer membrane protein (Omp2) gene is LI0902 of L. intracellularis. The homology of amino acid sequence between Omp2 and LI0902 is $99.1 \%$. Watson et al. used LC-ESI-MS/MS to analyze the two isolates of $L$. intracellularis from heavily infected epithelial cell cultures, and 19 proteins containing LI0902 were identified, and the function of Omp2 of L. intracellularis is thought to mediate peptidoglycan recognition and play an important role in protein-protein interactions via the vWF domain. In addition, the recombinant Omp2 protein could be recognized by sera from all but one of the infected groups and was not recognized by sera from any of the uninfected animals (33). Vannucci et al. used laser capture microdissection coupled with RNA-seq technology to characterize the transcriptional responses of infected eukaryotic cells and found that LI0902 was highly expressed in the cytoplasm of epithelial cells infected by L. intracellularis (34). Therefore, preparation of monoclonal antibodies against Omp2 is beneficial to research the pathogenic mechanism of L. intracellularis and monitor the L. intracellularis in infected intestinal epithelial cells.

L. intracellularis is an obligate intracellular bacterium that requires a specific microaerophilic environment and grows on intestinal epithelial cells $(1,35,36)$. Many cell lines, such as rat small intestinal cells (IEC-18), murine fibroblast-like (McCoy), human fetal intestine (INT 407), pig kidneys (PK15), and porcine jejunum cells (IPEC-J2), have been used to support the growth of $L$. intracellularis $(18,28)$. In this study, IEC-18 cells were used to culture L. intracellularis. To the best of our knowledge, the cells are easily contaminated by other bacteria in the intestine of pigs after inoculation with intestinal homogenate. Therefore, the isolation of $L$. intracellularis from clinical samples is extremely difficult (37). To date, there is no report regarding the successful isolation and maintenance of $L$. intracellularis infection from infected intestinal samples in China. In a previous study, L. intracellularis entered intestinal epithelial cells within $3 \mathrm{~h}$ after inoculation and was released from the vacuoles to subsequently live and proliferate in the apical cytoplasm (38). Our results also revealed that the infected cells could be precisely detected after being incubated for $3 \mathrm{~h}$ in a gas concentration of $8.0 \%$ $\mathrm{O}_{2}, 8.8 \% \mathrm{CO}_{2}$, and $83.2 \% \mathrm{~N}_{2}$. To obtain the high-quality intestines, it is necessary to screen the samples. Therefore, incubation for $3 \mathrm{~h}$ will improve the detection efficiency. Thus, in this study, to improve the detection efficiency, the infected samples were only cultured for $3 \mathrm{~h}$ after inoculation into IEC18 cells.

All the three monoclonal antibodies were specific to L. intracellularis and did not react with pure cultures of the enteric bacteria commonly found in the ileum of pigs or closely related to $L$. intracellularis. The 4D9, 3G2, and 7G5 MAbs detected L. intracellularis antigen in IEC-18 cells that were positive by IFA. The different intensities of staining by IFA using different antibodies were probably because of the titer of antibodies. Furthermore, L. intracellularis antigen was visualized mainly in the crypt of the ileum from infected tissues of PPE after it reacted with L. intracellularis-specific monoclonal antibody. These results demonstrated that the new monoclonal antibody could be applied to evaluate $L$. intracellularis in infected monolayer cells and tissues of PPE. This method will make an important contribution to monitoring of the L. intracellularis infection.

In conclusion, the recombinant Omp2 protein of $L$. intracellularis was successfully expressed in this study through prokaryotic expression technology. Western blot results showed that the protein could react with the positive serum of $L$. intracellularis. Subsequently, the recombinant Omp2 protein was used to immunize BALB/mice, and three monoclonal antibodies against Omp2 were screened by indirect ELISA, the monoclonal antibody specific against $L$. intracellularis will be useful for the evaluation of L. intracellularis infection in vivo and in vitro.

\section{DATA AVAILABILITY STATEMENT}

The datasets presented in this study can be found in online repositories. The names of the repository/repositories and accession number(s) can be found in the article/supplementary material.

\section{ETHICS STATEMENT}

The animal study was reviewed and approved by the Ethical Committee of the Faculty of Veterinary Science of Nanjing Agricultural University.

\section{AUTHOR CONTRIBUTIONS}

NX designed and drafted the work and wrote the manuscript. NX, JL, ML, YH, and HL performed the experiments, analyzed the data, and interpreted the results. HF helped conceive the project and edit the manuscript. All authors contributed to the article and approved the submitted version.

\section{FUNDING}

This study was supported by the National Key Research and Development Program of China (2017YFD0500203), 
the Jiangsu Agricultural Science and Technology Innovation Fund (CX(19)2020), the Industry-University Cooperation Program of Jiangsu Province (NBG0060265-1), and the Priority Academic Program Development of Jiangsu Higher Education Institutions (PAPD).

\section{REFERENCES}

1. Vannucci FA, Gebhart CJ. Recent advances in understanding the pathogenesis of Lawsonia intracellularis infections. Vet Pathol. (2014) 51:465-77. doi: 10.1177/0300985813520249

2. Leite FL, Abrahante JE, Vasquez E, Vannucci F, Gebhart CJ, Winkelman $\mathrm{N}$, et al. A cell proliferation and inflammatory signature is induced by Lawsonia intracellularis infection in swine. mBio. (2019) 10:e01605-18. doi: 10.1128/mBio.01605-18

3. Watarai M, Yoshiya M, Sato A, Furuoka H. Cultivation and characterization of Lawsonia intracellularis isolated from rabbit and pig. J Vet Med Sci. (2008) 70:731-3. doi: 10.1292/jvms.70.731

4. Pusterla N, Gebhart C. Lawsonia intracellularis infection and proliferative enteropathy in foals. Vet Microbio. (2013) 167:34-41. doi: 10.1016/j.vetmic.2013.06.017

5. Resende TP, Pereira CER, Daniel AGS, Vasquez E, Saqui-Salces M, Vannucci FA, et al. Effects of Lawsonia intracellularis infection in the proliferation of different mammalian cell lines. Vet Microbiol. (2019) 228:157-64. doi: 10.1016/j.vetmic.2018.11.029

6. Yang L, Lai F, He L, Lu Y, Zhong Q, Lai C, et al. LI1035, a putative effector secreted by Lawsonia intracellularis, targets the MAPK pathway and regulates actin organizationin yeast and mammalian cells. Vet Microbiol. (2019) 235:127-35. doi: 10.1016/j.vetmic.2019.06.009

7. Lawson GH, Gebhart CJ. Proliferative enteropathy. J Comp Pathol. (2000) 122:77-100. doi: 10.1053/jcpa.1999.0347

8. Koyama T, Hirai T, Nagai S. In vitro cultivation and partial characterization of Lawsonia intracellularis from a Japanese field case of porcine proliferative enteropathy. J Vet Med Sci. (2006) 68:609-13. doi: 10.1292/jvms.68.609

9. McOrist S. Defining the full costs of endemic porcine proliferative enteropathy. Vet J. (2005) 170:8-9. doi: 10.1016/j.tvjl.2004.04.008

10. Jensen HM. Health management with reduced antibiotic use experiences of a Danish pig vet. Anim Biotechnol. (2006) 17:189-94. doi: 10.1080/10495390600957142

11. Guedes RM, Gebhart CJ, Armbruster GA, Roggow BD. Serologic follow-up of a repopulated swine herd after an outbreak of proliferative hemorrhagic enteropathy. Can J Vet Res. (2002) 66:258-63.

12. McOrist S, Smits RJ. Field evaluation of an oral attenuated Lawsonia intracellularis vaccine for porcine proliferative enteropathy (ileitis). Vet Rec. (2007) 161:26-8. doi: 10.1136/vr.161.1.26

13. Park S, Lee JB, Kim KJ, Oh YS, Kim MO, Oh YR, et al. Efficacy of a commercial live attenuated Lawsonia intracellularis vaccine in a large scale field trial in Korea. Clin Exp Vaccine Res. (2013) 2:135-9. doi: 10.7774/cevr.2013.2.2.135

14. Lee SW, Kim TJ, Park SY, Song CS, Chang HK, Yeh JK, et al. Prevalence of porcine proliferative enteropathy and its control with tylosin in Korea. J Vet Sci. (2001) 2:209-12. doi: 10.4142/jvs.2001.2.3.209

15. Wu ZX, Ling Y, Tian DY, Pan Q, Heegaard PMH, He C. Seroprevalence of Lawsonia intracellularis antibodies in intensive pig farms in China. Bmc Vet Res. (2014) 10:100. doi: 10.1186/1746-6148-10-100

16. Kim J, Choi C, Cho WS, Chae C. Immunohistochemistry and polymerase chain reaction for the detection of Lawsonia intracellularis in porcine intestinal tissues with proliferative enteropathy. J Vet Med Sci. (2000) 62:7713. doi: $10.1292 /$ jvms.62.771

17. Wattanaphansak S, Pereira CER, Kaenson W, Assavacheep P, Tantilertcharoen $\mathrm{R}$, Resende $\mathrm{TP}$, et al. Isolation and in vitro antimicrobial susceptibility of porcine Lawsonia intracellularis from Brazil and Thailand. BMC Microbiol. (2019) 19:27. doi: 10.1186/s12866-019-1 397-7

18. Knittel JP, Larson DI, Harris D, Roof MB, McOrist S. United States isolates of Lawsonia intracellularis from porcine proliferative enteropathy resemble

\section{ACKNOWLEDGMENTS}

We would like to thank Changfeng Chen for assistance in obtaining the Omp2 protein. Furthermore, we wish to thank Yu Meng for the help in animal experiment.

European isolates. J Swine Health Prod. (1996) 4:119-22. Available online at: https://www.cabdirect.org/cabdirect/abstract/19962215666

19. Boye M, Jensen TK, Møller K, Leser TD, Jorsal SE. Specific detection of Lawsonia intracellularis in porcine proliferative enteropathy inferred from fluorescent rRNA in situ hybridization. Vet Pathol. (1998) 35:153-6. doi: 10.1177/030098589803500212

20. Hwang JM, Lee JH, Yeh JY. Generation of a monoclonal antibody against Mycoplasma spp. following accidental contamination during production of a monoclonal antibody against Lawsonia intracellularis. Appl Environ Microbiol. (2012) 78:2046-8. doi: 10.1128/aem.06705-11

21. Hoshino T, Yilmaz LS, Noguera DR, Daims H, Wagner M. Quantification of target molecules needed to detect microorganisms by fluorescence in situ hybridization (FISH) and catalyzed reporter deposition-FISH. Appl Environ Microbiol. (2008) 74:5068-77. doi: 10.1128/aem.00208-08

22. Aistleitner K, Jeske R, Wölfel R, Wießner A, Kikhney J, Moter A, et al. Detection of Coxiella burnetii in heart valve sections by fluorescence in situ hybridization. J Med Microbiol. (2018) 67:537-42. doi: 10.1099/jmm.0.000704

23. Guedes RM, Gebhart CJ, Winkelman NL, Mackie-Nuss RA. A comparative study of an indirect fluorescent antibody test and an immunoperoxidase monolayer assay for the diagnosis of porcine proliferative enteropathy. J Vet Diagn Invest. (2002) 14:420-3. doi: 10.1177/104063870201400512

24. Gao S, Shi W, Wang YT, Guo MT, Duan KX, Song AC, et al. Establishment and evaluation of an indirect immunofluorescence assay for the detection of salmonid alphavirus. Lett Appl Microbiol. (2018) 66:293-9. doi: 10.1111/lam.12834

25. Wang Q, Zhou H, Lin H, Ma Z, Fan H. Porcine circovirus type 2 exploits JNK-mediated disruption of tight junctions to facilitate Streptococcus suis translocation across the tracheal epithelium. Vet Res. (2020) 51:31. doi: 10.1186/s13567-020-00756-2

26. Ti J, Li Z, Li X, Lu Y, Diao Y, Li F. Identification of one B-cell epitope from NS1 protein of duck Tembusu virus with monoclonal antibodies. PLoS ONE. (2017) 12:e0181177. doi: 10.1371/journal.pone.0181177

27. Xiao N, Cao J, Zhou H, Ding SQ, Kong LY, Li JN. Identification of three novel B-cell epitopes of VMH protein from Vibrio mimicus by screening a phage display peptide library. Vet Immunol Immunopathol. (2016) 182:22-8. doi: 10.1016/j.vetimm.2016.09.005

28. Lawson GH, McOrist S, Jasni S, Mackie RA. Intracellular bacteria of porcine proliferative enteropathy: cultivation and maintenance in vitro. J Clin Microbiol. (1993) 31:1136-42. doi: 10.1128/jcm.31.5.1136-1142.1993

29. Roerink F, Morgan CL, Knetter SM, Passat MH, Archibald AL, AitAli $\mathrm{T}$, et al. A novel inactivated vaccine against Lawsonia intracellularis induces rapid induction of humoral immunity, reduction of bacterial shedding and provides robust gut barrier function. Vaccine. (2018) 36:1500-8. doi: 10.1016/j.vaccine.2017.12.049

30. Szczotka A, Stadejek T, Zmudzki J, Nowak A, Osiński Z, Pejsak Z. Immunohistochemical detection of Lawsonia intracellularis in tissue sections from pigs. Pol J Vet Sci. (2011) 14:531-8. doi: 10.2478/v10181-0 11-0079-7

31. McCluskey J, Hannigan J, Harris JD, Wren B, Smith DG. LsaA, an antigen involved in cell attachment and invasion, is expressed by Lawsonia intracellularis during infection in vitro and in vivo. Infect Immun. (2002) 70:2899-907. doi: 10.1128/iai.70.6.2899-2907.2002

32. Guedes RM, Gebhart CJ. Preparation and characterization of polyclonal and monoclonal antibodies against Lawsonia intracellularis. J Vet Diagn Invest. (2003) 15:438-46. doi: 10.1177/104063870301500506

33. Watson E, Alberdi MP, Inglis NF, Lainson A, Porter ME, Manson E, et al. Proteomic analysis of Lawsonia intracellularis reveals expression of outer membrane proteins during infection. Vet Microbiol. (2014) 174:448-55. doi: 10.1016/j.vetmic.2014.10.002 
34. Vannucci FA, Foster DN, Gebhart CJ. Laser microdissection coupled with RNA-seq analysis of porcine enterocytes infected with an obligate intracellular pathogen (Lawsonia intracellularis). Bmc Genomics. (2013) 14:421. doi: 10.1186/1471-2164-14-421

35. Karuppannan AK, Opriessnig T. Lawsonia intracellularis: revisiting the disease ecology and control of this fastidious pathogen in pigs. Front Vet Sci. (2018) 5:181. doi: 10.3389/fvets.2018.00181

36. Leite FL, Vasquez E, Gebhart CJ, Isaacson RE. The effects of Lawsonia intracellularis, Salmonella enterica serovar Typhimurium and co-infection on IL-8 and TNF $\alpha$ expression in IPEC-J2 cells. Vet Microbiol. (2019) 231:76-9. doi: 10.1016/j.vetmic.2019.02.036

37. Collins A. Advances in ileitis control, diagnosis, epidemiology and the economic impacts of disease in commercial pig herds. Agriculture. (2013) 3:536-55. doi: 10.3390/agriculture3030536

38. McOrist S, Jasni S, Mackie RA, Berschneider HM, Rowland AC, Lawson GH. Entry of the bacterium ileal symbiont intracellularis into cultured enterocytes and its subsequent release. Res Vet Sci. (1995) 59:255-60. doi: 10.1016/0034-5288(95)90013-6
Conflict of Interest: The authors declare that the research was conducted in the absence of any commercial or financial relationships that could be construed as a potential conflict of interest.

Publisher's Note: All claims expressed in this article are solely those of the authors and do not necessarily represent those of their affiliated organizations, or those of the publisher, the editors and the reviewers. Any product that may be evaluated in this article, or claim that may be made by its manufacturer, is not guaranteed or endorsed by the publisher.

Copyright (c) 2021 Xiao, Li, Li, Hu, Lin and Fan. This is an open-access article distributed under the terms of the Creative Commons Attribution License (CC BY).

The use, distribution or reproduction in other forums is permitted, provided the original author(s) and the copyright owner(s) are credited and that the original publication in this journal is cited, in accordance with accepted academic practice. No use, distribution or reproduction is permitted which does not comply with these terms. 